\title{
Probing Baryogenesis using Neutron-Anti-Neutron Oscillation
}

\author{
Kåre Fridell, ${ }^{*}$ Julia Harz and Chandan Hati \\ Physik Department T70, Technische Universität München, \\ James-Franck-Straße 1, 85748 Garching, Germany \\ E-mail: kare.fridell@tum.de
}

\begin{abstract}
Neutron-anti-neutron $(n-\bar{n})$ oscillation is a baryon number violating process that requires physics beyond the Standard model. Future experiments at ESS and DUNE aim to either discover $(n-\bar{n})$ oscillation or to put more stringent constraints on the oscillation time. We study the impact of such a discovery on different baryogenesis scenarios using an effective field theory framework for $(n-\bar{n})$ oscillation. We also study the implications of observing the mediators at the LHC and the possibility of falsifying some of the baryogenesis scenarios in the context of some simplified UV complete scenarios.
\end{abstract}

40th International Conference on High Energy physics - ICHEP2020

July 28 - August 6, 2020

Prague, Czech Republic (virtual meeting)

\footnotetext{
${ }^{*}$ Speaker
}

Preprint number: TUM-HEP-1300/20

(c) Copyright owned by the author(s) under the terms of the Creative Commons 


\section{Introduction}

Violation of baryon number $B$ is a requirement in any model that tries to explain the existence of a baryon asymmetry of the Universe. In order to have successful baryogenesis ${ }^{1}$, the three Sakharov conditions i) $B$ violation, ii) $\mathrm{C}$ and $\mathrm{CP}$ violation, and iii) out-of-equilibrium dynamics, need to be satisfied [1]. In the SM, the first condition is fulfilled by the sphaleron interactions, which violate $B+L$, the sum of baryon and lepton numbers. However, the sphaleron interaction is not enough for baryogenesis to occur. Therefore, in order to understand the baryon asymmetry of the Universe, it is necessary to search for additional sources of $B$ or $L$ violation. To this end, there are only a handful of ways to test $B$ or $L$ violation in a laboratory setting, some of the most prominent being neutron-anti-neutron oscillation, dinucleon decay, neutrinoless double beta decay, and proton decay.

Neutron-anti-neutron $(n-\bar{n})$ oscillation is an unobserved process that converts a neutron into an anti-neutron. This interaction violates conservation of baryon number $B$ by two units, $|\Delta B|=2$, and can not occur in the Standard Model (SM), thereby requiring New Physics (NP) beyond the SM (BSM). Experimental programs to constrain the neutron-anti-neutron oscillation time $\tau_{n-\bar{n}}$ involve bound searches (where the neutron is bound to a nuclei) and free searches (where the neutron is not bound). Currently, the most stringent constraint on the $n-\bar{n}$ oscillation time comes from the Super-Kamiokande experiment [2], which provides a limit $\tau_{n-\bar{n}}^{\text {bound }} \geq 4.7 \times 10^{8} \mathrm{~s}$. For the free $n-\bar{n}$ oscillation time, the most stringent constraint at $\tau_{n-\bar{n}}^{\text {free }} \geq 0.86 \times 10^{8} \mathrm{~s}$ comes from the ILL experiment [4]. Experimental limits on both the free and bound $n-\bar{n}$ oscillation times are expected to be improved in the near future. At the DUNE experiment, the limit on the bound $n-\bar{n}$ oscillation time is expected to reach $\tau_{n-\bar{n}}^{\text {bound }} \sim 7 \times 10^{8} \mathrm{~s}[3,5]$ while for the free oscillation, the NNBAR experiment at ESS is expected to provide the most stringent bound on the free $n-\bar{n}$ oscillation time of $\tau_{n-\bar{n}}^{\text {free }} \geq 3 \times 10^{9} \mathrm{~s}$ [5]. Due to the current stringent limits and expected improvements in the experimental sensitivities for the future $n-\bar{n}$ oscillation searches, it is very timely to study the impact of $n-\bar{n}$ oscillation on baryogenesis.

An observation of $n-\bar{n}$ oscillation would have implications for the matter/anti-matter asymmetry of the Universe. Out of the Sakharov conditions, $n-\bar{n}$ oscillation only probes $B$ violation, which by itself is not enough for baryogenesis, but may be connected to the other conditions through various BSM realizations of the $n-\bar{n}$ oscillation mechanism. Only fulfilling $B$ violation is, however, enough for a process to wash out a preexisting baryon asymmetry. In such a scenario, assuming baryogenesis to have occurred at a high energy scale, a washout of the matter/anti-matter asymmetry can transpire through the same mechanism that mediates the oscillation between neutron and anti-neutron, effectively reducing or removing the asymmetry completely. Therefore, constraining $n-\bar{n}$ oscillation parameters experimentally is crucial for understanding the generation of the baryon asymmetry of the Universe, and exploring the theoretical consequences of a potential $n-\bar{n}$ oscillation signal is an attractive gateway to discern possible NP models leading to such an asymmetry.

\footnotetext{
${ }^{1}$ Baryogenesis is the mechanism by which a baryon asymmetry is generated.
} 


\section{Neutron-anti-neutron effective field theory}

Assuming the NP that mediates the $n-\bar{n}$ oscillation to contain degrees of freedom which appear at a high energy scale as compared to the mass of a nucleon, the oscillation can be described by the effective Lagrangian

$$
\mathcal{L}_{\mathrm{eff}}^{\bar{n}-n}=\sum_{i=1,2,3,5}\left(C_{i}(\mu) O_{i}(\mu)+C_{i}^{P}(\mu) O_{i}^{P}(\mu)\right)+\text { h.c. } .
$$

Here, $\mu$ is the energy scale at which the operator is defined, and $C_{i}$ are effective Wilson coefficients that correspond to the effective dimension- 9 operators $O_{i}$. The relevant independent operators with non-vanishing matrix elements are given by [6]

$$
\begin{aligned}
& O_{1}=\left(\psi C P_{R} i \tau^{2} \psi\right)\left(\psi C P_{R} i \tau^{2} \psi\right)\left(\psi C P_{R} i \tau^{2} \tau^{+} \psi\right) T^{A A S}, \\
& O_{2}=\left(\psi C P_{L} i \tau^{2} \psi\right)\left(\psi C P_{R} i \tau^{2} \psi\right)\left(\psi C P_{R} i \tau^{2} \tau^{+} \psi\right) T^{A A S}, \\
& O_{3}=\left(\psi C P_{L} i \tau^{2} \psi\right)\left(\psi C P_{L} i \tau^{2} \psi\right)\left(\psi C P_{R} i \tau^{2} \tau^{+} \psi\right) T^{A A S}, \\
& O_{5}=\left(\psi C P_{R} i \tau^{2} \tau^{-} \psi\right)\left(\psi C P_{L} i \tau^{2} \tau^{+} \psi\right)\left(\psi C P_{L} i \tau^{2} \tau^{+} \psi\right) T^{S S S},
\end{aligned}
$$

where parity transformed operators (denoted by a superscript $P$ ) can be obtained by the replacement $P_{L} \leftrightarrow P_{R}$, and $\psi_{a}=(u, d)$ is a quark isospin doublet, with $u$ and $d$ being Dirac spinors. Furthermore, $P_{R, L}=\frac{1}{2}\left(1 \pm \gamma_{5}\right)$ are projection operators acting on the Lorentz-indices of the quark doublets, $\tau^{i}$ is the $i$ th Pauli matrix, such that $i \tau^{2}$ is the two-dimensional antisymmetric tensor acting on the isospin indices of the quark doublets, with $\tau^{ \pm}=\frac{1}{2}\left(\tau^{1} \pm i \tau^{2}\right)$, and $C$ is the charge conjugation matrix. Finally, $T^{S S S}$ and $T^{A S S}$ are symmetric and antisymmetric tensors respectively, given by

$$
\begin{aligned}
T_{\{i j\}\{k l\}\{m n\}}^{S S S} & =\varepsilon_{i k m} \varepsilon_{j l n}+\varepsilon_{j k m} \varepsilon_{i l n}+\varepsilon_{i l m} \varepsilon_{j k n}+\varepsilon_{i k n} \varepsilon_{j l m}, \\
T_{[i j][k l]\{m n\}}^{A A S} & =\varepsilon_{i j m} \varepsilon_{k l n}+\varepsilon_{i j n} \varepsilon_{k l m},
\end{aligned}
$$

which act on the color indices of the quark doublets. From the Lagrangian in Eq. (1), the $n-\bar{n}$ oscillation time $\tau_{n-\bar{n}}$ can be obtained to be given by

$$
\tau_{n-\bar{n}}^{-1}=\left\langle\bar{n}\left|\mathcal{L}_{\mathrm{eff}}^{\bar{n}-n}\right| n\right\rangle=\left|\sum_{i=1,2,3,5}\left(C_{i}(\mu) \mathcal{M}_{i}(\mu)+C_{i}^{P}(\mu) \mathcal{M}_{i}^{P}(\mu)\right)\right|,
$$

where $\mathcal{M}_{i}$ is the matrix element corresponding to operator $O_{i}$. In order to match the operators at the energy scale of $n-\bar{n}$ oscillations to the scale of new physics, which is assumed to be relatively high, renormalization group (RG) running effects should be taken into account. Defining the operators at a low energy scale $\mu_{0}$, which satisfies $m_{b}>\mu_{0}>m_{c}$, where $m_{b}$ and $m_{c}$ are the masses of the bottom and charm quarks respectively, their evolution to a higher energy scale $\mu_{\mathrm{NP}}>m_{t}$, where $m_{t}$ is the mass of the top quark, can be calculated as [7]

$$
O_{i}(\lambda)=U_{i}^{\prime}\left(\mu_{N P}, \mu_{0}\right) O_{i}\left(\mu_{0}\right) \equiv U_{i}^{N_{f}=6}\left(\mu_{N P}, m_{t}\right) U_{i}^{N_{f}=5}\left(m_{t}, m_{b}\right) U_{i}^{N_{f}=4}\left(m_{b}, \mu_{0}\right) O_{i}\left(\mu_{0}\right),
$$

where $N_{f}$ is the number of fermions, and $U_{i}^{N_{f}}\left(q_{1}, q_{2}\right)$ is given by [10]

$$
\begin{aligned}
U_{i}^{N f}\left(q_{1}, q_{2}\right)= & \left(\frac{\alpha_{S}\left(q_{2}\right)}{\alpha_{S}\left(q_{1}\right)}\right)^{-\gamma_{i}^{0} / 8 \pi \beta_{0}} \\
& \times\left[1-\delta_{q_{2}, \mu_{0}} r_{i}^{(0)} \frac{\alpha_{S}\left(\mu_{0}\right)}{4 \pi}+\left(\frac{\beta_{1} \gamma_{i}^{(0)} 4 \pi}{2 \beta_{0}^{2}}-\frac{\gamma_{i}^{(1)}}{2 \beta_{0}}\right) \frac{\alpha_{S}\left(q_{2}\right)-\alpha_{S}\left(q_{1}\right)}{16 \pi^{2}}\right] .
\end{aligned}
$$


Here $r^{(0)}, \gamma_{j}^{(i)}$ and $\beta_{i}$ are dimensionless constants, where $\gamma_{j}^{(i)}$ and $\beta_{i}$ depend on the number of fermions $N_{f}, \delta_{i j}$ is the Kronecker delta function, and $\alpha_{S}(\mu)$ is the strong coupling constant defined at an energy scale $\mu$. Taking values for the matrix elements $\mathcal{M}_{i}$ obtained using lattice quantum chromodynamics in [6], the operators are evolved to a high energy NP scale $\mu_{N P}$ where the Wilson coefficients $C_{i}$ are be defined. In this way, heavy NP can be related to $n-\bar{n}$ oscillation at the energy scale of the experiment, and a possible $n-\bar{n}$ oscillation signal can be understood in terms of the effective operators coming from high-mass BSM particles.

\section{Baryogenesis}

In order to study the effects on baryogenesis coming from a $n-\bar{n}$ operator, we use the Boltzmann equation $[8,9]$

$$
z H n_{\gamma} \frac{d \eta_{X}}{d z}=-\sum_{a, i, j, \cdots}[X a \cdots \leftrightarrow i j \cdots]
$$

which describes an evolution of the number density of a particle, in this case $X$, under the influence of scatterings and decays. Here $z=m_{X} / T$ is the variable under which the number density evolves, where $m_{X}$ is the mass of particle $X$ and $T$ is the temperature. Furthermore, $H$ is the Hubble rate, and $\eta_{X}=n_{X} / n_{\gamma}$ is the number density of particle $X$ normalized to the number density of photons $n_{\gamma}$, where the Hubble rate and photon number density are given by

$$
H=1.66 \sqrt{g_{*}} \frac{T^{2}}{\Lambda_{\mathrm{pl}}}, \quad n_{\gamma}=\frac{2 \zeta(3)}{\pi^{2}} T^{3},
$$

where $g_{*} \approx 107$ is the number of degrees of freedom, $\Lambda_{\mathrm{pl}} \approx 1.2 \times 10^{19} \mathrm{GeV}$ is the Planck scale, and $\zeta$ is the Riemann zeta function. The quantity in square brackets on the right-hand side of Eq. (7) is defined as

$$
[X a \cdots \leftrightarrow i j \cdots] \equiv \frac{n_{X} n_{a} \cdots}{n_{X}^{\mathrm{eq}} n_{a}^{\mathrm{eq}} \cdots} \gamma^{\mathrm{eq}}(X a \cdots \rightarrow i j \cdots)-\frac{n_{i} n_{j}}{n_{i}^{\mathrm{eq}} n_{j}^{\mathrm{eq}} \cdots} \gamma^{\mathrm{eq}}(i j \cdots \rightarrow X a \cdots),
$$

where $n_{i}^{\mathrm{eq}}$ is the number density in equilibrium of particle $i$, and $\gamma^{\mathrm{eq}}$ is the scattering density in thermal equilibrium. The sum in Eq. (7) ensures that all particle interactions in which $X$ participates are involved in the evolution of the number density. Under the influence of a washout process ${ }^{2}$, without any mechanism to generate an asymmetry ${ }^{3}$, Eq. (7) can be re-expressed in terms of density of baryon number $\Delta B$,

$$
z H n_{\gamma} \frac{d \eta_{\Delta B}}{d z}=-\Gamma^{\mathrm{w}} T^{3} \eta_{\Delta B}
$$

where $\Gamma^{\mathrm{w}}$ is the rate of the washout process. With Eq. (10), a baryon number violating interaction such as $n-\bar{n}$ oscillation can be connected to the washout of baryon asymmetry in the early

\footnotetext{
${ }^{2}$ Washout is a mechanism by which a baryon asymmetry is reduced or removed via baryon number violating interactions.

${ }^{3}$ Here it is assumed that the asymmetry was already generated at a higher temperature.
} 


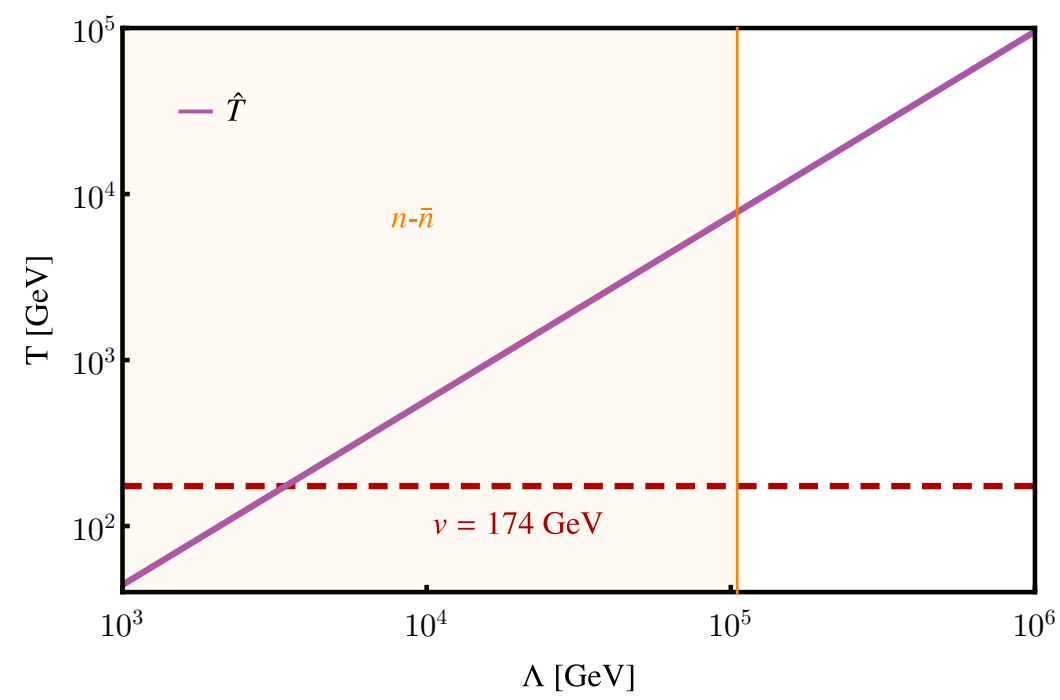

Figure 1: Temperature $T_{D}(\Lambda)$ at which the fraction $\Gamma / H$ is equal to one (purple solid line), as a function of the NP scale $\Lambda$. The red dashed line shows the scale of electroweak symmetry breaking.

Universe. As an example, focusing on the $n-\bar{n}$ oscillation operator $O_{5}$ from Eq. (2), using the methods described in [7,11], the equilibrium scattering rate is obtained as

$$
\gamma_{O_{5}}^{\mathrm{eq}}=\frac{25}{\pi^{9}} \frac{T^{14}}{C_{5}(T)^{2}} .
$$

This leads to a washout rate

$$
\Gamma_{O_{5}}^{\mathrm{W}}=\frac{1375}{21 \pi^{9}} \frac{T^{11}}{C_{5}(T)^{2}}
$$

In Fig 1, the temperature $\hat{T}$ at which the fraction $\Gamma_{O_{5}}^{\mathrm{w}} / H$ is equal to one is shown as a function of the NP scale $\Lambda$. Above the temperature $\hat{T}$, the washout coming from operator $O_{5}$ can be assumed to be strong [7]. The current experimental reach of $n-\bar{n}$ oscillation experiments is shown in shaded orange, as calculated using Eq. (4) for the limit $\tau_{n-\bar{n}}^{\text {bound }} \geq 4.7 \times 10^{8} \mathrm{~s}$ coming from the Super-Kamiokande experiment [2]. A more accurate calculation can be performed by integrating the relevant Boltzmann equations [7]. Furthermore, in the presence of $\mathrm{CP}$ violating interactions involving new fields which violate baryon number and induce $n-\bar{n}$ oscillation, a complete and consistent treatment of the Boltzmann equations involving the decays and washout processes is necessary to test the viability of high- and low-scale baryogenesis mechanisms [7].

\section{Summary}

Neutron-anti-neutron oscillation will be searched for at an unprecedented sensitivity in the coming years. An observation of such a $B$ violating NP process as $n-\bar{n}$ would open the door for answers to many outstanding problems in particle physics, providing a clear sign of NP beyond the SM, and shedding light on the origin of the matter/anti-matter asymmetry in the Universe. In the case of a non-observation on the other hand, the upcoming exclusion limits on the $n-\bar{n}$ oscillation time will put severe restrictions on the parameter space of viable baryogenesis models, since many 
of them predict $n-\bar{n}$ oscillation at an observable rate. Therefore, improving the experimental lower limit on the $n-\bar{n}$ oscillation time could contribute to falsifying various baryogenesis models, and would provide a step closer to understanding the overabundance of matter in comparison to anti-matter in the Universe.

\section{Acknowledgments}

The authors acknowledge support from the DFG Emmy Noether Grant No. HA 8555/1-1.

\section{References}

[1] A. D. Sakharov, Sov. Phys. Usp. 34 (1991) no.5, 392-393 doi:10.1070/PU1991v034n05ABEH002497

[2] K. Abe et al. [Super-Kamiokande], [arXiv:2012.02607 [hep-ex]].

[3] R. Acciarri et al. [DUNE], [arXiv:1512.06148 [physics.ins-det]].

[4] M. Baldo-Ceolin, P. Benetti, T. Bitter, F. Bobisut, E. Calligarich, R. Dolfini, D. Dubbers, P. El-Muzeini, M. Genoni and D. Gibin, et al. Z. Phys. C 63 (1994), 409-416 doi:10.1007/BF01580321

[5] A. Addazi, K. Anderson, S. Ansellb, K. S. Babua, J. Barrow, D. V. Baxter, P. M. Bentley, Z. Berezhiani, R. Bevilacqua and R. Biondi, et al. [arXiv:2006.04907 [physics.ins-det]].

[6] E. Rinaldi, S. Syritsyn, M. L. Wagman, M. I. Buchoff, C. Schroeder and J. Wasem, Phys. Rev. D 99 (2019) no.7, 074510 doi:10.1103/PhysRevD.99.074510 [arXiv:1901.07519 [hep-lat]].

[7] K. Fridell, J. Harz and C. Hati, In preparation.

[8] K. Griest and D. Seckel, Phys. Rev. D 43 (1991), 3191-3203 doi:10.1103/PhysRevD.43.3191

[9] G. F. Giudice, A. Notari, M. Raidal, A. Riotto and A. Strumia, Nucl. Phys. B 685 (2004), 89-149 doi:10.1016/j.nuclphysb.2004.02.019 [arXiv:hep-ph/0310123 [hep-ph]].

[10] M. I. Buchoff and M. Wagman, Phys. Rev. D 93 (2016) no.1, 016005 [erratum: Phys. Rev. D 98 (2018) no.7, 079901] doi:10.1103/PhysRevD.93.016005 [arXiv:1506.00647 [hep-ph]].

[11] F. F. Deppisch, L. Graf, J. Harz and W. C. Huang, Phys. Rev. D 98 (2018) no.5, 055029 doi:10.1103/PhysRevD.98.055029 [arXiv:1711.10432 [hep-ph]]. 